\title{
Review Article \\ Custom-Made Antibiotic Cement Nails in Orthopaedic Trauma: Review of Outcomes, New Approaches, and Perspectives
}

\author{
Marcin K. Wasko' ${ }^{1}$ and Rafal Kaminski ${ }^{2}$ \\ ${ }^{1}$ Department of Orthopaedics and Rheumoortopaedics, Prof. A. Gruca Teaching Hospital, Konarskiego 13, 05-400 Otwock, Poland \\ ${ }^{2}$ Department of Trauma Surgery and Orthopaedics, Prof. A. Gruca Teaching Hospital, Konarskiego 13, 05-400 Otwock, Poland
}

Correspondence should be addressed to Marcin K. Wasko; m.wasko@cmkp.edu.pl

Received 20 February 2015; Revised 18 May 2015; Accepted 20 May 2015

Academic Editor: Francesco Piraino

Copyright ( 2015 M. K. Wasko and R. Kaminski. This is an open access article distributed under the Creative Commons Attribution License, which permits unrestricted use, distribution, and reproduction in any medium, provided the original work is properly cited.

\begin{abstract}
Since the first description in 2002 by Paley and Herzenberg, antibiotic bone cement nails (ACNs) have become an effective tool in the orthopaedic trauma surgeons' hands. They simultaneously elute high amounts of antibiotics into medullary canal dead space and provide limited stability to the debrided long bone. In this paper, we perform a systematic review of current evidence on ACNs in orthopaedic trauma and provide an up-to-date review of the indications, operative technique, failure mechanisms, complications, outcomes, and outlooks for the ACNs use in long bone infection.
\end{abstract}

\section{Introduction}

Musculoskeletal infections remain a challenge for orthopaedic surgeons and infectious disease specialists. Bone provides a unique milieu for bacteria, with low vascularity and turnover rate. Most of the orthopaedic trauma infections are caused by biofilm-forming bacteria [1]. Biofilm consists of hydrated matrix of polysaccharide and protein. Once formed, it protects the microorganism from antimicrobials, opsonization, and phagocytosis, thus contributing to the chronicity of infections [2]. In order to cure biofilm-related infection, four principles formulated by Cierny and Mader must be observed: (a) complete surgical debridement with dead space management, (b) fracture/nonunion stabilization, (c) soft tissue coverage, and $(\mathrm{d})$ adequate antibiotic levels [3]. In healthy bone, local antibiotics' concentrations might be less than $20 \%$ of serum levels, as is for most beta-lactams [4]. Their efficacy is further diminished by biofilms, which decrease molecule penetration [5]. With intramedullary infections, the optimal way of debridement is to ream the medullary canal. After reaming, it takes approximately 4 weeks for bone to revascularise [6]. Therefore, even with prolonged antibiotic therapy, local bone tissue remains without bactericidal concentrations, thus not interfering with bacterial growth.
Acrylic bone cement is the gold standard for dead space management and the standard carrier for local antibiotic delivery in the management of orthopaedics infections $[2,7]$. It delivers high concentrations of the drug locally, even to avascular areas that are inaccessible to systemic antibiotics. Those concentrations are high enough to be effective even against organisms that are resistant to drug concentrations achieved by intravenous supply. At the same time, very low serum antibiotic concentrations are observed and hence the risk for toxicity is considerably diminished $[8,9]$.

Antibiotic loaded bone cement can be customized intraoperatively to different shapes and forms. In intramedullary infections, antibiotic bone cement nails or antibiotic cement nails (ACNs) are preferable. Figure 1 presents an example of an ACN. They offer local delivery of antibiotics, while filling the dead space and offering stability to the fracture/nonunion site, if present.

The primary objective of this paper is to perform a systematic review of current evidence on ACNs in orthopaedic trauma. The secondary objective was to provide an up-todate review of the indications, operative technique, failure mechanisms, complications, outcomes, and outlooks for the ACNs use in musculoskeletal infection. 


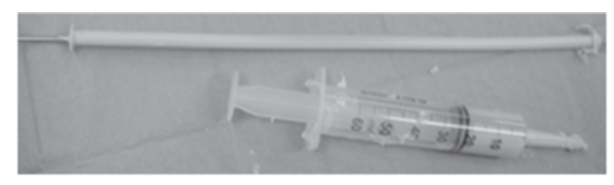

FIGURE 1: The photo of the prepared intraoperatively $K$-wire-armed, antibiotic loaded cement nail with a syringe (for scale comparison).

\section{Materials and Methods}

2.1. Search Strategy. To identify relevant papers, we searched Medline database via PubMed interface with no restriction on language or publication date. The search string included word "intramedullary" and one of the following words, "osteomyelitis," "infection," "nail," "nailing," "debridement," and "reaming" and was performed on January 1, 2015. We searched all fields in the Medline database, with no restriction applied to full-text availability. Additionally, we manually reviewed the reference lists of the articles retrieved by database search for potentially missing papers.

The clinical studies selected were original articles on antibiotic cement nails. We excluded all the studies on bone cement use in arthroplasty and spine surgery as well as in vitro and in vivo studies, although we did not use the "restrict to human studies" filter in PubMed.

Details such as the number of patients, anatomical site, the age of the patients, the preparation and composition of nail, length of follow-up, and final outcome were collected.

\section{Results and Discussion}

3.1. Search Results. Literature review of antibiotic cement nails cases is given in Table 1. Figure 2 shows search strategy flow diagram. Since detailed discussion of clinical outcomes is beyond the scope of this review, for detailed discussion of clinical outcomes and perspectives on intramedullary infection treatment, including ACNs, we suggest the reader consults an excellent clinical review by Makridis et al. [10].

3.2. Indications for ACNs. The indication for the use of $\mathrm{ACN}$ is medullary infection, whatever the cause and presentation. Intramedullary infection is a well-recognized complication of intramedullary nailing for trauma [11]. It spreads along the length of the nail and involves the entire length of bone [12]. Multiple points or also the entire medullary canal is involved in pin tract infections after external fixation [13]. Reported rates of infectious complications after planned conversion from external fixation to intramedullary nailing for the femoral fractures range from 1.7 to $20 \%$ [14-18]. Similar problems can occur with lengthening over nails with external fixation and transport over nail $[19,20]$. Therefore, the indications for ACN span from long bone fractures with concomitant soft tissue damage, to infected nonunion sequelae of external fixation and haematogenous osteomyelitis.

All these diagnoses share a common trait; there is usually no sequestrum and dead bone is limited to within the medullary canal [21]. However, according to the Cierny principles, after the removal of the intramedullary nail it acts as an avascular noncollapsible dead space that needs to be managed [22]. It could not be managed appropriately with poly(methyl methacrylate) (PMMA) beads strung on elastic wire and introduced into medullary canal, since they do not conform to the dead space shape and their removal becomes increasingly difficult starting already 2 weeks after insertion due to fibrous overgrowth [23]. Rather than using multiple beads on a single wire, Klemm and in another paper Seligson and Klemm used PMMA stick, formed of a PMMA mass attached to a single wire [24, 25]. This construct could be passed around external fixator's pins and its removal was facilitated. Unfortunately, it did not provide any mechanical stability to the fracture/nonunion site. In that scenario, stability is very important to treat the infection and to obtain drainage cessation or lessening $[26,27]$.

3.3. Contraindications for ACNs. There is one paper stating that, in bone deficits exceeding $6 \mathrm{~cm}$, other alternatives for restoring stability and infection control should be used [34]. There is also an obvious contraindication for the reaming and, thus, the use of nail in people under 16 years of age [28].

3.4. The Role of Local Antibiotics Delivery versus the Role of Debridement. In a 2013 Cochrane review, the authors could not show any significant difference in osteomyelitis recurrence rates after parenteral or oral antibiotic administration [39]. Although it might be due to the fact that only limited and low quality evidence on the subject is available, this might also suggest the importance of surgical debridement as a cure, which is an observation commonly accepted among the orthopaedic surgeons [40]. A prospective trial by Simpson et al. documented the effect of surgical debridement on cure rates. They achieved $100 \%$ success with wide excision (clearance margin of $5 \mathrm{~mm}$ or more), $28 \%$ failure rate with marginal resection, and a total failure with local debulking and intralesional biopsy [41]. The importance of this issue is highlighted by articles describing novel ways to thoroughly debride the medullary canal, for example, RIA or Pressure Sentinel [42]. Therefore, it must be borne in mind that all the clinical results of ACNs show at the same time the results of debridement and other therapeutic actions [43].

3.5. Nail Fabrication. Multiple techniques for the fabrication of the nails have been described, from manual rolling of the cement [34], through the use of chest tube as a temporary mould, which is peeled off once the cement hardens [12, 21, $23,29,35,37,38,44]$, to using a reusable mould $[45,46]$.

Usually, a chest tube or another kind of drainage tube with an inner diameter similar to the outer diameter of the removed nail or the diameter of the last reamer used is selected and closed at one end, for example, with Kocher forceps. Some kind of guide wire ( $K$-wire, Ender nail, etc.) is selected and cut a little bit longer than the tube and its end is bent to facilitate later extraction from the medullary canal. It can also be bent to adapt to the dead space, for example, with the Herzog angle in case of replacing a tibia nail. Later, antibiotic powder is mixed with poly(methyl methacrylate) powder. The next step is to add liquid monomer, usually 


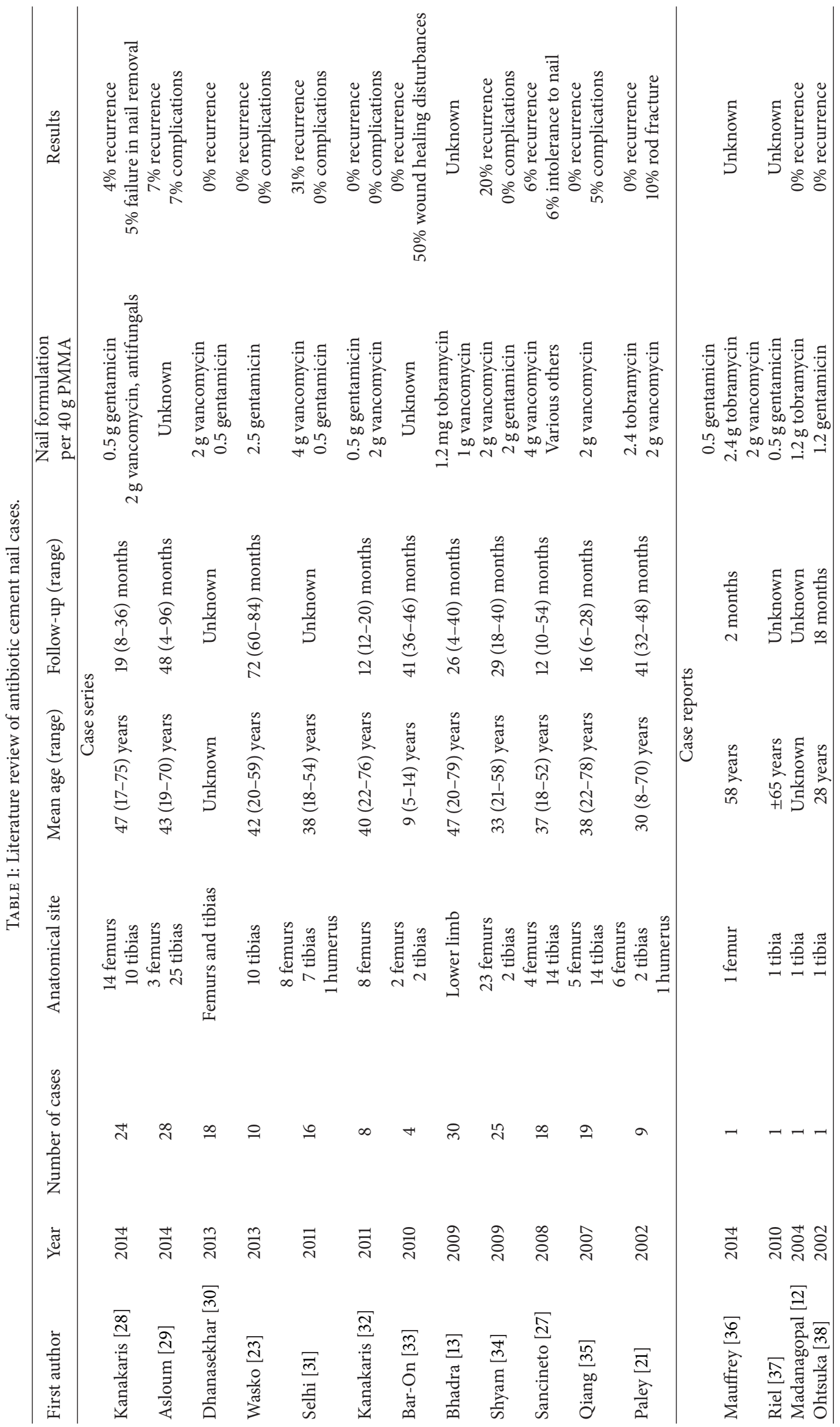




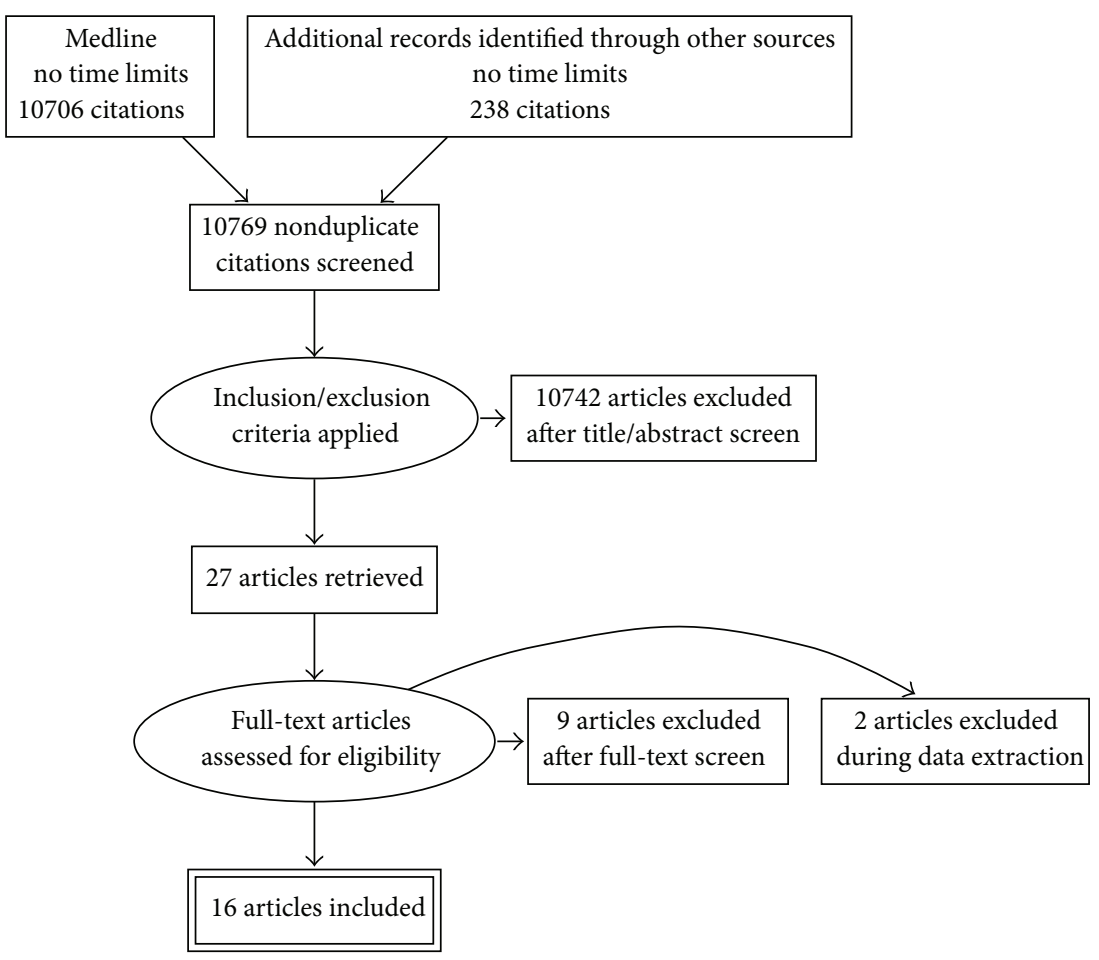

FIGURE 2: Article search strategy flow diagram for this paper.

more than would be used for the single batch of cement, to compensate for large volume of antibiotics. Next, the cement is poured into the cement gun and injected into the tube. The precut wire is inserted into the middle of the cement in the tube. At some point, either when the cement begins to harden and heats up or after the exothermic reaction, the tube is cut with surgical knife and peeled from the cement. Then the surgeon waits for the nail to cool and for the monomer to evaporate, which usually takes around 15 minutes. The nail can then be inserted into the medullary canal, with the bent/looped end extruding for easier retrieval.

3.6. Cement Type. When compared with other bone cements, Palacos cement showed highest elution rates, which was explained by its inherent porosity [48].

3.7. Mixing Techniques. It has been advocated that vacuum mixing the cement results in stable antibiotic elution [21], although this effect varies for different cement types [49]. On the other hand, there are papers arguing that vacuum mixing decreases cement's porosity and thus reduces the total elution of the antibiotic [50]. In-depth analysis was provided by Neut et al., who showed that vacuum-mixed gentamicinloaded Palacos $\mathrm{R}$ released more gentamicin after one week in vitro than hand-mixed Palacos $\mathrm{R}$ did despite a reduction in the porosity, which theoretically should reduce elution [51]. The authors explained this discrepancy by the increase in the number and distribution of micropores smaller than one millimetre in the vacuum-mixing group, which occurred during cement polymerization by evaporation of the volatile monomer. On the other hand, the same article mentions that hand mixing with a spatula resulted in increased antibiotic release than hand mixing in a dedicated system (Cemvac) [51]. It has also been brought to attention that mixing systems in general are very heterogeneous in regard to resulting cement porosity, which probably influences the elution rates a great deal $[52,53]$.

\subsection{Antibiotics}

3.8.1. Antibiotics: General Considerations. To effectively eliminate bacteria in a biofilm, local antibiotic concentrations achieved must be 10 to 100 times the usual bactericidal concentration [5]. This usually cannot be achieved by safe doses of parenteral antibiotics, rendering this form of biofilm treatment ineffective [2]. Bone cement can deliver high concentrations of antibiotics, even to poorly vascularised and hypoxic environment, as it is independent of vascular supply $[21,54-56]$. For the most popular antibiotics, bactericidal concentrations were found for up to six weeks after implanting PMMA beads [57]. Most likely, this observation would be applicable to the use of nails, since hip spacers show similar pharmacokinetics [58].

The antibiotics used for the fabrication of ACNs should be available in powder form and have a wide antibacterial spectrum with bactericidal activity at low concentrations [59]. Other desirable characteristics include high elution rate from PMMA over long periods of time and thermal stability with low influence for the mechanical properties of the cement [8]. Since they are eluted locally, they should not cause allergy or bind to serum protein easily, to maintain high 
concentration $[48,54]$. They should also have as minimal as possible inhibitory influence on new bone formation [60].

Liquid antibiotics elute larger amounts from bone cement than antibiotics in powder form [8]. However, they are not used in clinical practice since they negatively influence the mechanical properties of PMMA [61, 62]. Antibiotics in powder form are reported to have a rather negligible effect on the mechanical stability of bone cement as long as the antibiotic to cement ratio does not exceed $10 \%$, which is a lower proportion than usually applied in the ACNs formulation [63].

3.8.2. Antibiotic Choice: Microorganism Sensibility. Nowadays, effective serum levels describe sensitivity of bacteria to the antibiotics. Most bacteria defined as resistant by these criteria might be sensitive when exposed to local antibiotics $[7,55,64]$. To guide the antimicrobial treatment properly, pathogens should be reconsidered to be either sensitive or resistant to the antibiotic levels achievable locally $[7,31$, 55]. This is the case, for example, in classical Buchholz's work, where the antibiotic placed in the bone cement did not necessarily correlate with the culture-based sensitivity of the organisms [65]. On the other hand, there have been reports of changing patterns in microorganism resistance, partly as a result of widespread local and systemic prophylaxis [66]. Increasing numbers of gentamicin-resistant species are reported to cause deep infections, including medullary infections $[67,68]$. One must remember that, even with antibiotics' concentrations as high as after adding up to $20 \%$ weight of the PMMA, colonization of the spacer/nail can still occur [69].

3.8.3. Antibiotics: Dosage. Acrylic bone cement was primarily developed as a fixation device for arthroplasty. Although the addition of more than two grams of any antibiotic per $40 \mathrm{~g}$ of cement reduces the cement's strength, this is not relevant to the infection treatment, as those devices are only temporary and only partially loaded mechanically [56]. To maintain elution rates and concentrations sufficient to treat an established musculoskeletal infection, at least $3.4 \mathrm{~g}$ of antibiotic should be used for $40 \mathrm{~g}$ batch of PMMA [70, 71].

3.8.4. Antibiotics: Elution. There are no papers on antibiotic elution from ACNs in English. The only report we were able to find was a Japanese article with no English translation [72]. However, there is a multitude of antibiotic elution studies on cement beads and spacers. Although the absolute values for drug release from ACNs might be different from beads and spacers, the general proportions between elution rates and processes governing the elution remain the same [73].

The first study on elution was performed by Marks et al., who showed that oxacillin, cefazolin, and gentamicin elute in a microbiologically active form from Palacos and Simplex bone cements [62]. Afterwards, it was established that elution of antibiotics from acrylic cement follows a biphasic pattern, with high elution rates early, followed by slower but sustained elution rates as time progresses [8]. Only a small portion of the antibiotic incorporated in bone cement is released; the amount of antibiotic released from cement shows an exponential decline after day 1 of implantation $[49,51,74]$. The hydrophobicity of ALBC permits roughly $10 \%$ of the added antibiotic to elute over a 6-8-week period [8].

The mechanism by which these drugs are released is not fully understood. First papers to tackle that issue suggested that the elution of antibiotics from bone cement was mainly by diffusion $[75,76]$. The diffusion theory relies on the presence of pores and interconnected channels in bone cement, through which the circulating medium penetrates and dissolves the incorporated antibiotics which then slowly diffuse outwards [77]. However, Masri et al. argued that the data provided by the diffusion theory protagonists did not support this conclusion [78].

Nowadays, most of the research suggests that antibiotic release from PMMA is a passive phenomenon in which diffusion occurs out of pores, cracks, and voids in the cement $[8,79]$. In support of this theory, studies show that elution is improved with increasing surface area and porosity of the cement. [62, 78-80]. van de Belt et al. studied the release of gentamicin as a function of time from six different gentamicin-loaded bone cements. They related elution to surface roughness, porosity, and wettability of the PMMA. The release kinetics of gentamicin in their study was controlled by surface roughness and porosity. They suggested that the initial release antibiotic from bone cement was mainly a surface phenomenon, while sustained release over several days was a bulk diffusion phenomenon [81]. This theory best accounts for the biphasic release characteristics of antibiotic bone cement [8].

The elution of antibiotic from PMMA would be therefore dependent on multiple variables: which cement is used [48, 54], which antibiotic is chosen [71], the amount of total antibiotic added [71], and how it is mixed [82]. Highly porous cement has been shown to elute more antibiotic and for a longer period of time relative to cement with less porosity [71].

Commercially manufactured antibiotic loaded drug delivery systems have more predictable elution patterns, compared to devices manufactured with a manual addition of an antibiotic (due to the more homogenous distribution of the incorporated antibiotic(s) in the cement powder). However, this is often the only way to make the bone cement appropriate to the sensitivity profile of the causative pathogen. Adding another antibiotic powder not only increases the activity spectrum but also increases the antibiotic elution rates. Most studies evaluating combinations of antibiotics have demonstrated a synergistic effect, in that adding a second antibiotic seems to increase the elution of both antibiotics $[8,54,71,83]$. This is true for tobramycin and vancomycin [71, 84], teicoplanin and gentamicin [83], and linezolid and gentamicin [85]. However, two studies have reported that combining antibiotics results in decreased elution [86, 87]. No change in elution rate was reported when vancomycin and amikacin had been used together; elution rates were the same as when used individually [73]. On the other hand, with the use of another PMMA cement, different antibiotics ratio, and different testing conditions, one can find that release of one of the drugs is inhibited by the addition of another antibiotic [87]. Generally, when two 
antibiotics are mixed into the same batch of bone cement, a phenomenon called passive opportunisms occurs; one of the substances acts as a soluble additive increasing porosity of the cement and increasing the total elution [71]. Thus, total antimicrobial effect of eluted substances is increased. This phenomenon seems to depend on the volume ratio between two antibiotics added to PMMA [8].

Díez-Peña et al. tried to predict the gentamicin elution from low- and high-loaded bone cement (containing up to $10 \mathrm{wt} \%$ of gentamicin sulfate and more than $20 \mathrm{wt} \%$ of gentamicin sulfate in relation to the weight of entire block of bone cement) by means of mathematical equations in an experimental model. They found out that each differently loaded PMMA has a different equation describing the release process. Moreover, for low-loaded cement, the release was mainly controlled by the imperfections of the matrix, whereas in the high-loaded PMMA an abrupt increase in the amount of drug released was evident allowing the almost complete release of the drug incorporated [88].

Lastly, the method used to mix the antibiotic seems to play an important role. Hand mixing with a spatula has been shown to increase the total antibiotic release when compared with mixing with a specialized cement mixer [51]. It is hypothesized that hand mixing introduces significant porosity to the cement, which in turn should increase antibiotic elution [8]. Other authors argue that hand mixing, in contrast to device mixing, does not crush the antibiotic crystals and thus may improve elution characteristic [50].

3.8.5. Antibiotics: Current Practice. The most used antibiotic for the fabrication of ACNs is gentamicin, followed by vancomycin $[30,44]$. Concomitant use of these two agents can widen the spectrum of activity but also enhance the elution rates for both substances simultaneously [89].

3.8.6. Antibiotics: Complications. Only low serum concentrations and minor systemic toxicity are achieved while using ACNs and other local cement delivery devices [54, 90]. Research has consistently shown that antibiotics added to cement devices do not reach significant concentrations in the bloodstream, and there is no systemic toxicity in otherwise healthy individuals without hepatic or renal disease [55]. We found four case reports that have been published on acute renal failure after the use of high-dose ALBC for the treatment of deep periprosthetic sepsis, three total knees and two total hip patients [91-94]. Concurrent administration of IV antibiotics and significant comorbidities (e.g., preexisting renal disease) complicate all of these patient histories. On the other hand, a study from Mayo clinic by Springer et al. which retrospectively reviewed 36 knees in 34 patients treated with $4 \mathrm{~g}$ of vancomycin and $4.8 \mathrm{~g}$ of gentamicin per $40 \mathrm{~g}$ batch of PMMA reported only a single case of transient elevation in serum creatinine with no permanent systemic complications [95]. Noteworthily, the mean total dose of antibiotic per patient in that study was $10.5 \mathrm{~g}$ of vancomycin and $12.5 \mathrm{~g}$ of gentamicin, which is much higher than with any of the ACNs reported. Up to date, we were not able to find any report on renal or auditory complication in ACN patients. This might be due to the fact that the majority of those nails are put in trauma or posttraumatic patients, who tend to be younger and previously healthy compared to, for example, total joint replacement patient population. Multiple papers show that patients treated with local antibiotics are at no more risk and are probably at less risk of experiencing ototoxicity and nephrotoxicity than ones subjected to long-term parenteral antibiotics [96-98]. There are more adverse reactions due to the use of systemic antibiotics than to the use of local antibiotic-eluting spacers and nails [99].

Another important complication of ACNs is resistance induction. There are two articles describing ability of bacteria to adhere to and colonize gentamicin [69] and mixed gentamicin-vancomycin-loaded cement [100] after prolonged periods of implantation and even ability to develop gentamicin resistance despite preoperative susceptibility.

3.9. In Vitro and In Vivo Studies on ACNs. As far as the authors know, there is no report on the use of ACNs in an animal model. Most probably this is due to the technical difficulties and availability of similar models, that is, antibioticcoated implants. Local application of a biodegradable coating with $10 \%$ gentamicin was shown to be effective in reducing implant-related infection in a rat model [101]. Similar data was found for fibrin sealant plus tobramycin, a combination which was as effective as poly(methyl methacrylate) beads plus tobramycin against methicillin-sensitive Staphylococcus aureus osteomyelitis in a rabbit model [102].

Elson et al. performed one of the most important early laboratory studies. They showed that when antibiotic loaded acrylic bone cement is placed next to cortical bone, dense cortical bone is penetrated by the eluted antibiotic and its concentration in the bone is much higher than what can be achieved safely by systemic administration [103].

3.10. ACNs Benefits and Risks. The benefits and risks of ACNs are summarised in Table 2 . Since the benefits were thoroughly discussed in the previous sections of this paper, only ACNs' shortcomings are described below.

First and foremost, local antibiotic carriers have never been shown to be superior to intravenous administration of antibiotics in terms of cure rate. At the same time, most of them require some sort of repeat surgery, unless they biodegrade, which is not the case in ACNs [44].

There has been one report linking high failure rate to the use of ACNs in infected nonunion with bone defect exceeding $6 \mathrm{~cm}$ [34]. The authors suggested that when faced with a large bone defect, other alternative forms of treatment should be used. Unfortunately, they did not provide a multivariate analysis on risk factors that might influence their results.

One of the concerns with all the PMMA carriers for antibiotic is the emergence of resistance. There have been reports on induction of coagulase-negative staphylococci after applying gentamicin-loaded bone cement [69]. However, there are, to our knowledge, no reports on resistance after ACNs.

From the technical point of view, there is a possibility of debonding of the cement from the nail upon its introduction or removal from the medullary canal. However, this situation 
TABLE 2: The benefits and drawbacks of antibiotic cement nails.

Benefits

High concentration of local antibiotic elution: up to 200 times greater than with systemic drug administration, independent of vascular supply [21] Stability to the fracture/nonunion site, allowing for early weight bearing [23] Local antibiotic delivery independent of patient compliance [23] Systemic toxicity of antibiotics very rarely observed [8] Versatility of modifying antibiotic as per the culture report [27] Control of infection and stability is achieved with a single procedure [44] Alternative for patients refusing or not being right candidates for external fixation [13]
Drawbacks

Local antibiotic carriers have never been shown to be superior to intravenous administration of antibiotics in terms of cure rate [21]

Require repeat surgery [23]

Possible emergence of resistance [13]

Possible MMA toxicity $[102,103]$ is usually quickly resolved with reaming or using longreaching surgical tools and, in our experience, as with every surgical complication, the rate of debonding decreases with the familiarity with the technique and increasing expertise in nail fabrication $[23,46]$.

In the past, bone cement liquid monomer, methyl methacrylate (MMA), was believed to be carcinogenic. However, the lack of consistency in the results of various studies and the absence of dose response lead to the conclusion that MMA in not carcinogenic to humans under normal conditions of use [104]. Moreover, the evidence shows that even repeated mixing of PMMA bone cement does not pose an additional risk to operative theatres' personnel [105]. In a study comparing ionically dissolved or precipitated metals with MMA toxicity, MMA monomer toxicity was found to be low compared to metal toxicity [47]. The study of Elmaraghy et al. found that the presence of MMA monomer in femoral venous blood has no effect on the formation of fat emboli or their pulmonary haemodynamic outcome during cemented hip arthroplasty [106]. To our knowledge, there are no studies on MMA toxicity after the use of ACNs.

3.11. Alternatives. The novel concept of local antibiotic delivery by gentamicin-impregnated acrylic bone cement was introduced by Buchholz et al. in the early 1970s for the treatment of infected hip arthroplasty [65]. Antibiotic bead chains were introduced by Klemm in 1976 [24]. Later on, his team reported on the use of an antibiotic-impregnated cement "stick" $[25,107]$.

In a study of two cases, Tandon and Thomas were able to provide a proof of concept of using hollow, slotted nail with gentamicin cement beads. Based on the observation that commercially available beads are $7 \mathrm{~mm}$ in diameter, they argued that two to three strings of beads could be inserted into most intramedullary nails and effectively treat infection [108]. Unfortunately, their technique did not allow for the use of interlocking screws, as they would make extracting the beads without the nail impossible, and was never published on a larger series.

3.12. Outlook. It is perceived that with future developments local antibiotic delivery systems will likely supplant the traditional use of systemic antibiotics for the treatment of musculoskeletal infections [7, 109].

Another field of development is the MRI-compatible ACN. There has been only a single, short follow-up case report by Mauffrey et al. The authors claim that serum inflammatory markers might not always be reliable and that MRI monitoring of the infection might provide better insight into whether or not the infection has healed and one could move to the definitive fixation by a metallic nail [36]. On the other hand, the use of carbon fibre nail was estimated to incur an additional cost of $\$ 2,600$, which is almost 30 -fold higher than for most of the nails described in the literature [23].

Since 2005, there are also prefabricated interlocking antibiotic-coated titanium nails, which use polylactic acid (PLA) coating loaded with gentamicin and offer both sustained release kinetics and biodegradability. Promising 6month results have been reported in primary fracture setting [110].

It is important to realize that as long as none of the ACNs is FDA-approved, which is the current state as of 2015, their use is off-label and prospective clinical trials cannot be undertaken [44].

There is only one short paediatric case series, in which ACNs have been used. It was found to be safe and effective in the treatment of chronic osteomyelitis, with special attention to protecting the epiphyseal growth plates of long bones. The authors believed also that using the nail instead of other forms of local antibiotic carrier enabled for prevention of further tissue loss [33]. We believe this patient group could benefit from the use of ACNs, but further clinical trials are necessary before introducing the ACNs into regular paediatric trauma/orthopaedic practice.

There are certain substances other than antibiotics, such as dextran, glycine, or xylitol, which could be used to impregnate PMMA for the enhancement of the antibiotic elution. However, the ideal filler material and amount of filler are yet to be established [8]. We are not aware of any studies beyond the laboratory phase on the PMMA fillers [111].

Another technical development is the introduction of the Reamer-Irrigator-Aspirator (RIA) system (Synthes, Inc., West Chester, Philadelphia) which, in animal models and cadaver studies, offers an advance on existing reaming devices, as it is less traumatic [32, 42]. It is used in the debridement and irrigation of the intramedullary canal of the femur and tibia [112]. Its use is very promising and could facilitate proper debridement of the medullary canal, which is the basic condition of intramedullary infection healing.

Ultrasound was also found to increase the transport of gentamicin across and within the biofilm of $P$. aeruginosa and E. coli. The other effect of ultrasound waves is presumably to 
increase the transport of oxygen and other small molecules, which may increase the metabolic state and render cells more susceptible to antibiotics $[113,114]$. The ultrasound may have also a positive effect on antibiotic elution characteristics [8]. Further laboratory, feasibility, and clinical studies are needed to explain the potential for enhanced antibiotic release from PMMA by ultrasound and translate those basic science findings into clinical practice.

\section{Conclusions}

Since the introduction of antibiotic loaded acrylic bone cement by Buchholz and Engelbrecht, it remains a golden standard in local antibiotic delivery. This is also true in case of long bone medullary infections, where antibiotic cement nails remain an important treatment option. The major advantage of ACNs is the local release of high antibiotic concentrations, which vastly exceed those after systemic administration with no or low systemic toxicity. At the same time, they are able to provide limited stability to the fracture/nonunion site, which promotes infection healing. They are used as a clinician directed application; therefore, there is no issue with availability and antibiotic specificity, as it can be based on preoperative culture. Antibiotic elution rates, mechanisms, and ways to improve total amount of drug delivered are still debated and researched. Despite promising short- and midterm results from clinical studies, further basic science and translational studies are desirable before routine use of ACNs in clinical practice.

\section{Conflict of Interests}

The authors declare that there is no conflict of interests regarding the publication of this paper.

\section{References}

[1] P. Stoodley, G. D. Ehrlich, P. P. Sedghizadeh et al., "Orthopaedic biofilm infections," Current Orthopaedic Practice, vol. 22, no. 6, pp. 558-563, 2011.

[2] C. L. Nelson, "The current status of material used for depot delivery of drugs," Clinical Orthopaedics and Related Research, no. 427, pp. 72-78, 2004.

[3] G. Cierny and J. Mader, "The surgical treatment of adult osteomyelitis," in Surgery of the Musculoskeletal System, pp. 4814-4834, Churchill Livingstone, New York, NY, USA, 1983.

[4] P. D. P. Lew and P. F. A. Waldvogel, "Osteomyelitis," The Lancet, vol. 364, no. 9431, pp. 369-379, 2004.

[5] P. S. Stewart and J. W. Costerton, "Antibiotic resistance of bacteria in biofilms," The Lancet, vol. 358, no. 9276, pp. 135-138, 2001.

[6] L. Lazzarini, J. T. Mader, and J. H. Calhoun, "Osteomyelitis in long bones," The Journal of Bone and Joint Surgery-American Volume, vol. 86, no. 10, pp. 2305-2318, 2004.

[7] A. D. Hanssen, "Local antibiotic delivery vehicles in the treatment of musculoskeletal infection," Clinical Orthopaedics and Related Research, no. 437, pp. 91-96, 2005.

[8] K. Anagnostakos and J. Kelm, "Enhancement of antibiotic elution from acrylic bone cement," Journal of Biomedical Materials
Research Part B: Applied Biomaterials, vol. 90, no. 1, pp. 467-475, 2009.

[9] S. B. Rosslenbroich, M. J. Raschke, C. Kreis et al., "Daptomycin: local application in implant-associated infection and complicated osteomyelitis," The Scientific World Journal, vol. 2012, Article ID 578251, 9 pages, 2012.

[10] K. Makridis, T. Tosounidis, and P. Giannoudis, "Suppl 2: management of infection after intramedullary nailing of long bone fractures: treatment protocols and outcomes," The Open Orthopaedics Journal, vol. 7, pp. 219-226, 2013.

[11] M. J. Patzakis, J. Wilkins, and D. A. Wiss, "Infection following intramedullary nailing of long bones: diagnosis and management," Clinical Orthopaedics and Related Research, vol. 212, pp. 182-191, 1986.

[12] S. G. Madanagopal, D. Seligson, and C. S. Roberts, "The antibiotic cement nail for infection after tibial nailing," Orthopedics, vol. 27, no. 7, pp. 709-712, 2004.

[13] A. K. Bhadra and C. S. Roberts, "Indications for antibiotic cement nails," Journal of Orthopaedic Trauma, vol. 23, supplement 5, pp. S26-S30, 2009.

[14] P. J. Nowotarski, C. H. Turen, R. J. Brumback, and J. M. Scarboro, "Conversion of external fixation to intramedullary nailing for fractures of the shaft of the femur in multiply injured patients," The Journal of Bone \& Joint Surgery-American Volume, vol. 82, no. 6, pp. 781-788, 2000.

[15] T. M. Scalea, S. A. Boswell, J. D. Scott, K. A. Mitchell, M. E. Kramer, and A. N. Pollak, "External fixation as a bridge to intramedullary nailing for patients with multiple injuries and with femur fractures: damage control orthopedics," The Journal of Trauma-Injury, Infection and Critical Care, vol. 48, no. 4, pp. 613-623, 2000.

[16] A. A. Parekh, W. R. Smith, S. Silva et al., "Treatment of distal femur and proximal tibia fractures with external fixation followed by planned conversion to internal fixation," Journal of Trauma-Injury, Infection and Critical Care, vol. 64, no. 3, pp. 736-739, 2008.

[17] M. Bernat, C. Lecoq, M. Lempidakis, G. Martin, R. Aswad, and D. G. Poitout, "Secondary internal osteosynthesis after external fixation for recent or old open fracture of the lower limb," Revue de Chirurgie Orthopédique et Réparatrice de L'Appareil Moteur, vol. 82, no. 2, pp. 137-144, 1996.

[18] D. J. Maurer, R. L. Merkow, and R. B. Gustilo, "Infection after intramedullary nailing of severe open tibial fractures initially treated with external fixation," The Journal of Bone \& Joint Surgery-American Volume, vol. 71, no. 6, pp. 835-838, 1989.

[19] D. Paley, J. E. Herzenberg, and N. Bor, "Fixator-assisted nailing of femoral and tibial deformities," Techniques in Orthopaedics, vol. 12, no. 4, pp. 260-275, 1997.

[20] G. Oedekoven, D. Jansen, M. Raschke, and B. F. Claudi, "The monorail system-bone segment transport over unreamed interlocking," Der Chirurg, vol. 67, no. 11, pp. 1069-1079, 1996.

[21] D. Paley and J. E. Herzenberg, "Intramedullary infections treated with antibiotic cement rods: preliminary results in nine cases," Journal of Orthopaedic Trauma, vol. 16, no. 10, pp. 723729, 2002.

[22] K. Tetsworth and G. Cierny III, "Osteomyelitis debridement techniques," Clinical Orthopaedics and Related Research, no. 360, pp. 87-96, 1999.

[23] M. K. Wasko and O. Borens, "Antibiotic cement nail for the treatment of posttraumatic intramedullary infections of the 
tibia: midterm results in 10 cases," Injury, vol. 44, no. 8, pp. 10571060, 2013.

[24] K. Klemm, "Gentamicin-PMMA-beads in treating bone and tissue infections (author's transl)," Zentralblatt für Chirurgie, vol. 104, no. 14, pp. 934-942, 1979.

[25] D. Seligson and K. Klemm, "Treatment of infection following intramedullary nailing," in The Science and Practice of Intramedullary Nailing, vol. 2, pp. 317-333, Lippincott Williams \& Wilkins, 1996.

[26] M. E. Miller, J. R. Ada, and L. X. Webb, "Treatment of infected nonunion and delayed union of tibia fractures with locking intramedullary nails," Clinical Orthopaedics and Related Research, no. 245, pp. 233-238, 1989.

[27] C. F. Sancineto and J. D. Barla, "Treatment of long bone osteomyelitis with a mechanically stable intramedullar antibiotic dispenser: nineteen consecutive cases with a minimum of 12 months follow-up," The Journal of Trauma-Injury, Infection and Critical Care, vol. 65, no. 6, pp. 1416-1420, 2008.

[28] N. Kanakaris, S. Gudipati, T. Tosounidis, P. Harwood, S. Britten, and P. V. Giannoudis, "The treatment of intramedullary osteomyelitis of the femur and tibia using the Reamer-IrrigatorAspirator system and antibiotic cement rods," The Bone \& Joint Journal, vol. 96-B, no. 6, pp. 783-788, 2014.

[29] Y. Asloum, G. Vergnenegre, B. Bedin et al., "L’enclouage cimenté transitoire en traumatologie. À propos de 26 cas," Revue de Chirurgie Orthopédique et Traumatologique, vol. 100, no. 4, pp. S91-S98, 2014.

[30] R. Dhanasekhar, P. Jacob, and J. Francis, "Antibiotic cement impregnated nailing in the management of infected non-union of femur and tibia," Kerala Journal of Orthopaedics, vol. 26, no. 2, pp. 93-97, 2013.

[31] H. S. Selhi, P. Mahindra, M. Yamin, D. Jain, W. G. De Long Jr., and J. Singh, "Outcome in patients with an infected nonunion of the long bones treated with a reinforced antibiotic bone cement rod," Journal of Orthopaedic Trauma, vol. 26, no. 3, pp. 184-188, 2012.

[32] N. K. Kanakaris, D. Morell, S. Gudipati, S. Britten, and P. V. Giannoudis, "Reaming irrigator aspirator system: early experience of its multipurpose use," Injury, vol. 42, no. 4, pp. S28-S34, 2011.

[33] E. Bar-On, D. M. Weigl, N. Bor et al., "Chronic osteomyelitis in children: treatment by intramedullary reaming and antibioticimpregnated cement rods," Journal of Pediatric Orthopaedics, vol. 30 , no. 5, pp. 508-513, 2010.

[34] A. K. Shyam, P. K. Sancheti, S. K. Patel, S. Rocha, C. Pradhan, and A. Patil, "Use of antibiotic cement-impregnated intramedullary nail in treatment of infected non-union of long bone," Indian Journal of Orthopaedics, vol. 43, no. 4, pp. 396-402, 2009.

[35] Z. Qiang, P. Z. Jun, X. J. Jie, L. Hang, L. J. Bing, and L. F. Cai, "Use of antibiotic cement rod to treat intramedullary infection after nailing: preliminary study in 19 patients," Archives of Orthopaedic and Trauma Surgery, vol. 127, no. 10, pp. 945-951, 2007.

[36] C. Mauffrey, G. W. Chaus, N. Butler, and H. Young, "MRcompatible antibiotic interlocked nail fabrication for the management of long bone infections: first case report of a new technique," Patient Safety in Surgery, vol. 8, no. 1, article 14, 2014.

[37] R. U. Riel and P. B. Gladden, "A simple method for fashioning an antibiotic cement-coated interlocking intramedullary nail," The American Journal of Orthopedics, vol. 39, no. 1, pp. 18-21, 2010.
[38] H. Ohtsuka, K. Yokoyama, K. Higashi et al., "Use of antibioticimpregnated bone cement nail to treat septic nonunion after open tibial fracture," Journal of Trauma-Injury, Infection and Critical Care, vol. 52, no. 2, pp. 364-366, 2002.

[39] L. O. Conterno and M. D. Turchi, "Antibiotics for treating chronic osteomyelitis in adults," The Cochrane Database of Systematic Reviews, vol. 9, Article ID CD004439, 2013.

[40] J. Sanders and C. Mauffrey, "Long bone osteomyelitis in adults: fundamental concepts and current techniques," Orthopedics, vol. 36, no. 5, pp. 368-375, 2013.

[41] A. H. R. W. Simpson, M. Deakin, and J. M. Latham, "Chronic osteomyelitis. The effect of the extent of surgical resection on infection-free survival," The Journal of Bone \& Joint SurgeryBritish Volume, vol. 83, no. 3, pp. 403-407, 2001.

[42] G. Goplen, J. A. Wilson, M. McAffrey, K. Deluzio, and R. Leighton, "A cadaver model evaluating femoral intramedullary reaming: a comparison between new reamer design (Pressure Sentinel) and a novel suction/irrigation reamer (RIA)," Injury, vol. 41, no. 2, pp. S38-S42, 2010.

[43] Y. Xu, Y. Zhu, X. Fan, T. Jin, Y. Li, and X. He, "Implantrelated infection in the Tibia: surgical revision strategy with vancomycin cement," The Scientific World Journal, vol. 2014, Article ID 124864, 6 pages, 2014.

[44] J. W. Kim, D. O. Cuellar, J. Hao, D. Seligson, and C. Mauffrey, "Custom-made antibiotic cement nails: a comparative study of different fabrication techniques," Injury, vol. 45, no. 8, pp. 11791184, 2014.

[45] R. Thonse and J. Conway, "Antibiotic cement-coated interlocking nail for the treatment of infected nonunions and segmental bone defects," Journal of Orthopaedic Trauma, vol. 21, no. 4, pp. 258-268, 2007.

[46] R. Thonse and J. D. Conway, "Antibiotic cement-coated nails for the treatment of infected nonunions and segmental bone defects," The Journal of Bone \& Joint Surgery-American Volume, vol. 90, no. 4, pp. 163-174, 2008.

[47] M. G. Shettlemore and K. J. Bundy, "Assessment of dental material degradation product toxicity using a bioluminescent bacterial assay," Dental Materials, vol. 18, no. 6, pp. 445-453, 2002.

[48] M. J. Penner, C. P. Duncan, and B. A. Masri, “The in vitro elution characteristics of antibiotic-loaded CMW and Palacos-R bone cements," Journal of Arthroplasty, vol. 14, no. 2, pp. 209-214, 1999.

[49] J. Meyer, G. Piller, C. A. Spiegel, S. Hetzel, and M. Squire, "Vacuum-mixing significantly changes antibiotic elution characteristics of commercially available antibiotic-impregnated bone cements," The Journal of Bone \& Joint Surgery-American Volume, vol. 93, no. 22, pp. 2049-2056, 2011.

[50] T. A. Clyburn and Q. Cui, "Antibiotic laden cement: current state of the art," AAOS Now, 2007.

[51] D. Neut, H. van de Belt, J. R. van Horn, H. C. van der Mei, and H. J. Busscher, "The effect of mixing on gentamicin release from polymethylmethacrylate bone cements," Acta Orthopaedica Scandinavica, vol. 74, no. 6, pp. 670-676, 2003.

[52] H. Mau, K. Schelling, C. Heisel, J.-S. Wang, and S. J. Breusch, "Comparison of various vacuum mixing systems and bone cements as regards reliability, porosity and bending strength," Acta Orthopaedica Scandinavica, vol. 75, no. 2, pp. 160-172, 2004. 
[53] A. Bistolfi, G. Massazza, E. Verné et al., "Antibiotic-loaded cement in orthopedic surgery: a review," ISRN Orthopedics, vol. 2011, Article ID 290851, 8 pages, 2011.

[54] D. Cerretani, G. Giorgi, P. Fornara et al., "The in vitro elution characteristics of vancomycin combined with imipenemcilastatin in acrylic bone-cements: a pharmacokinetic study," The Journal of Arthroplasty, vol. 17, no. 5, pp. 619-626, 2002.

[55] W. W. Brien, E. A. Salvati, R. Klein, B. Brause, and S. Stern, "Antibiotic impregnated bone cement in total hip arthroplasty: an in vivo comparison of the elution properties of tobramycin and vancomycin," Clinical Orthopaedics and Related Research, no. 296, pp. 242-248, 1993.

[56] M. Baleani, C. Persson, C. Zolezzi, A. Andollina, A. M. Borrelli, and D. Tigani, "Biological and biomechanical effects of vancomycin and meropenem in acrylic bone cement," The Journal of Arthroplasty, vol. 23, no. 8, pp. 1232-1238, 2008.

[57] D. Seligson, G. J. Popham, K. Voos, S. L. Henry, and M. Faghri, "Antibiotic-leaching from polymethylmethacrylate beads," The Journal of Bone \& Joint Surgery-American Volume, vol. 75, no. 5, pp. 714-720, 1993.

[58] E. Bertazzoni Minelli, A. Benini, B. Magnan, and P. Bartolozzi, "Release of gentamicin and vancomycin from temporary human hip spacers in two-stage revision of infected arthroplasty," The Journal of Antimicrobial Chemotherapy, vol. 53, no. 2, pp. 329-334, 2004.

[59] K. Anagnostakos, O. Fürst, and J. Kelm, "Antibiotic-impregnated PMMA hip spacers: current status," Acta Orthopaedica, vol. 77, no. 4, pp. 628-637, 2006.

[60] C. G. Zalavras, M. J. Patzakis, and P. Holtom, "Local antibiotic therapy in the treatment of open fractures and osteomyelitis," Clinical Orthopaedics and Related Research, no. 427, pp. 86-93, 2004.

[61] E. Ger, D. Dall, T. Miles, and A. Forder, "Bone cement and antibiotics," South African Medical Journal, vol. 51, no. 9, pp. 276-279, 1977.

[62] K. E. Marks, C. L. Nelson, and E. P. Lautenschlager, "Antibioticimpregnated acrylic bone cement," The Journal of Bone \& Joint Surgery-American Volume, vol. 58, no. 3, pp. 358-364, 1976.

[63] E. P. Lautenschlager, J. J. Jacobs, G. W. Marshall, and P. R. Meyer Jr., "Mechanical properties of bone cements containing large doses of antibiotic powders," Journal of Biomedical Materials Research, vol. 10, no. 6, pp. 929-938, 1976.

[64] A. D. Hanssen and M. J. Spangehl, "Practical applications of antibiotic-loaded bone cement for treatment of infected joint replacements," Clinical Orthopaedics and Related Research, no. 427, pp. 79-85, 2004.

[65] H. W. Buchholz, R. A. Elson, E. Engelbrecht, H. Lodenkämper, J. Röttger, and A. Siegel, "Management of deep infection of total hip replacement," The Journal of Bone \& Joint Surgery -British Volume, vol. 63, no. 3, pp. 342-353, 1981.

[66] P. Anguita-Alonso, A. D. Hanssen, D. R. Osmon, A. Trampuz, J. M. Steckelberg, and R. Patel, "High rate of aminoglycoside resistance among staphylococci causing prosthetic joint infection," Clinical Orthopaedics and Related Research, no. 439, pp. 43-47, 2005.

[67] C.-E. Chen, J.-Y. Ko, J.-W. Wang, and C.-J. Wang, "Infection after intramedullary nailing of the femur," The Journal of Trauma, vol. 55, no. 2, pp. 338-344, 2003.

[68] M. M. Tunney, G. Ramage, S. Patrick, J. R. Nixon, P. G. Murphy, and S. P. Gorman, "Antimicrobial susceptibility of bacteria isolated from orthopedic implants following revision hip surgery,"
Antimicrobial Agents and Chemotherapy, vol. 42, no. 11, pp. 3002-3005, 1998.

[69] B. Thornes, P. Murray, and D. Bouchier-Hayes, "Development of resistant strains of Staphylococcus epidermidis on gentamicinloaded bone cement in vivo," The Journal of Bone and Joint Surgery-British Volume, vol. 84, no. 5, pp. 758-760, 2002.

[70] W. A. Jiranek, A. D. Hanssen, and A. S. Greenwald, "Current concepts review: antibiotic-loaded bone cement for infection prophylaxis in total joint replacement," The Journal of Bone \& Joint Surgery-American Volume, vol. 88, no. 11, pp. 2487-2500, 2006.

[71] M. J. Penner, B. A. Masri, and C. P. Duncan, "Elution characteristics of vancomycin and tobramycin combined in acrylic bonecement," The Journal of Arthroplasty, vol. 11, no. 8, pp. 939-944, 1996.

[72] M. Tonegawa, A. Hashimoto, and M. Sekiguchi, "Release of gentamicin from acrylic bone cement spacer," Kotsu Kansetsu Jintai, vol. 11, pp. 1019-1024, 1998.

[73] D. K. Kuechle, G. C. Landon, D. M. Musher, and P. C. Noble, "Elution of vancomycin, daptomycin, and amikacin from acrylic bone cement," Clinical Orthopaedics and Related Research, no. 264, pp. 302-308, 1991.

[74] N. Greene, P. D. Holtom, C. A. Warren et al., "In vitro elution of tobramycin and vancomycin polymethylmethacrylate beads and spacers from Simplex and Palacos," American Journal of Orthopedics, vol. 27, no. 3, pp. 201-205, 1998.

[75] H. W. Buchholz, R. A. Elson, and K. Heinert, "Antibiotic-loaded acrylic cement: current concepts," Clinical Orthopaedics and Related Research, vol. 190, pp. 96-108, 1984.

[76] R. Bayston and R. D. G. Milner, "The sustained release of antimicrobial drugs from bone cement. An appraisal of laboratory investigations and their significance," The Journal of Bone \& Joint Surgery -British Volume, vol. 64, no. 4, pp. 460-464, 1982.

[77] H. van de Belt, D. Neut, W. Schenk, J. R. Van Horn, H. C. Van der Mei, and H. J. Busscher, "Infection of orthopedic implants and the use of antibiotic-loaded bone cements," Acta Orthopaedica Scandinavica, vol. 72, no. 6, pp. 557-571, 2001.

[78] B. A. Masri, C. P. Duncan, C. P. Beauchamp, N. J. Paris, and J. Arntorp, "Effect of varying surface patterns on antibiotic elution from antibiotic-loaded bone cement," The Journal of Arthroplasty, vol. 10, no. 4, pp. 453-459, 1995.

[79] A. S. Baker and L. W. Greenham, "Release of gentamicin from acrylic bone cement. Elution and diffusion studies," The Journal of Bone and Joint Surgery-American Volume, vol. 70, no. 10, pp. 1551-1557, 1988.

[80] B. M. Wroblewski, "Leaching out from acrylic bone cement. Experimental evaluation," Clinical Orthopaedics and Related Research, no. 124, pp. 311-312, 1977.

[81] H. van de Belt, D. Neut, D. R. A. Uges et al., "Surface roughness, porosity and wettability of gentamicin-loaded bone cements and their antibiotic release," Biomaterials, vol. 21, no. 19, pp. 1981-1987, 2000.

[82] G. Lewis, S. Janna, and A. Bhattaram, "Influence of the method of blending an antibiotic powder with an acrylic bone cement powder on physical, mechanical, and thermal properties of the cured cement," Biomaterials, vol. 26, no. 20, pp. 4317-4325, 2005.

[83] K. Anagnostakos, J. Kelm, T. Regitz, E. Schmitt, and W. Jung, "In vitro evaluation of antibiotic release from and bacteria growth inhibition by antibiotic-loaded acrylic bone cement spacers," Journal of Biomedical Materials Research Part B: Applied Biomaterials, vol. 72, no. 2, pp. 373-378, 2005. 
[84] B. A. Masri, C. P. Duncan, and C. P. Beauchamp, "Long-term elution of antibiotics from bone-cement: an in vivo study using the prosthesis of antibiotic-loaded acrylic cement (PROSTALAC) system," The Journal of Arthroplasty, vol. 13, no. 3, pp. 331338, 1998 .

[85] K. Anagnostakos, J. Kelm, S. Grün, E. Schmitt, W. Jung, and S. Swoboda, "Antimicrobial properties and elution kinetics of linezolid-loaded hip spacers in vitro," Journal of Biomedical Materials Research-Part B Applied Biomaterials, vol. 87, no. 1, pp. 173-178, 2008.

[86] M. E. Bertazzoni, C. Caveiari, and A. Benini, "Release of antibiotics from polymethylmethacrylate cement," Journal of Chemotherapy, vol. 14, no. 5, pp. 492-500, 2002.

[87] J. Klekamp, J. M. Dawson, D. W. Haas, D. DeBoer, and M. Christie, "The use of vancomycin and tobramycin in acrylic bone cement: biomechanical effects and elution kinetics for use in joint arthroplasty," The Journal of Arthroplasty, vol. 14, no. 3, pp. 339-346, 1999.

[88] E. Díez-Peña, G. Frutos, P. Frutos, and J. M. Barrales-Rienda, "Gentamicin sulphate release from a modified commercial acrylic surgical radiopaque bone cement. I. Influence of the gentamicin concentration on the release process mechanism," Chemical \& Pharmaceutical Bulletin, vol. 50, no. 9, pp. 12011208, 2002.

[89] K.-H. Koo, J.-W. Yang, S.-H. Cho et al., "Impregnation of vancomycin, gentamicin, and cefotaxime in a cement spacer for two-stage cementless reconstruction in infected total hip arthroplasty," The Journal of Arthroplasty, vol. 16, no. 7, pp. 882892, 2001.

[90] M. Kruger-Franke, C. Carl, and J. Haus, "Treatment of infection following intramedullary nailing. A comparison of various treatment regimens," Aktuelle Traumatologie, vol. 23, no. 2, pp. 72-76, 1993.

[91] J. M. Curtis, V. Sternhagen, and D. Batts, "Acute renal failure after placement of tobramycin-impregnated bone cement in an infected total knee arthroplasty," Pharmacotherapy, vol. 25, no. 6, pp. 876-880, 2005.

[92] S. Dovas, V. Liakopoulos, L. Papatheodorou et al., "Acute renal failure after antibiotic-impregnated bone cement treatment of an infected total knee arthroplasty," Clinical Nephrology, vol. 69, no. 3, pp. 207-212, 2008.

[93] B. N. Patrick, M. P. Rivey, and D. R. Allington, "Acute renal failure associated with vancomycin- and tobramycin-laden cement in total hip arthroplasty," The Annals of Pharmacotherapy, vol. 40, no. 11, pp. 2037-2042, 2006.

[94] T. M. van Raaij, L. E. Visser, A. G. Vulto, and J. A. N. Verhaar, "Acute renal failure after local gentamicin treatment in an infected total knee arthroplasty," The Journal of Arthroplasty, vol. 17, no. 7, pp. 948-950, 2002.

[95] B. D. Springer, G.-C. Lee, D. Osmon, G. J. Haidukewych, A. D. Hanssen, and D. J. Jacofsky, "Systemic safety of highdose antibiotic-loaded cement spacers after resection of an infected total knee arthroplasty," Clinical Orthopaedics and Related Research, no. 427, pp. 47-51, 2004.

[96] D. E. Stabile and A. M. Jacobs, "Development and application of antibiotic-loaded bone cement beads," Journal of the American Podiatric Medical Association, vol. 80, no. 7, pp. 354-359, 1990.

[97] K. Klemm, "The use of antibiotic-containing bead chains in the treatment of chronic bone infections," Clinical Microbiology and Infection, vol. 7, no. 1, pp. 28-31, 2001.
[98] R. C. Haydon, J. D. Blaha, C. Mancinelli, and K. Koike, "Audiometric thresholds in osteomyelitis patients treated with gentamicin- impregnated methylmethacrylate beads (Septopal)," Clinical Orthopaedics and Related Research, no. 295, pp. 43-46, 1993.

[99] J. D. Blaha, J. H. Calhoun, C. L. Nelson et al., "Comparison of the clinical efficacy and tolerance of gentamicin PMMA beads on surgical wire versus combined and systemic therapy for osteomyelitis," Clinical Orthopaedics and Related Research, no. 295, pp. 8-12, 1993.

[100] K. Anagnostakos, P. Hitzler, D. Pape, D. Kohn, and J. Kelm, "Persistence of bacterial growth on antibiotic-loaded beads: is it actually a problem?” Acta Orthopaedica, vol. 79, no. 2, pp. 302307, 2008.

[101] M. Lucke, G. Schmidmaier, S. Sadoni et al., "Gentamicin coating of metallic implants reduces implant-related osteomyelitis in rats," Bone, vol. 32, no. 5, pp. 521-531, 2003.

[102] J. T. Mader, C. M. Stevens, J. H. Stevens, R. Ruble, J. T. Lathrop, and J. H. Calhoun, "Treatment of experimental osteomyelitis with a fibrin sealant antibiotic implant," Clinical Orthopaedics and Related Research, no. 403, pp. 58-72, 2002.

[103] R. A. Elson, A. E. Jephcott, D. B. McGechie, and D. Verettas, "Antibiotic loaded acrylic cement," The Journal of Bone and Joint Surgery-British Volume, vol. 59, no. 2, pp. 200-205, 1977.

[104] J. A. Tomenson, A. V. Carpenter, and M. A. Pemberton, "Critical review of the epidemiology literature on the potential cancer risks of methyl methacrylate," International Archives of Occupational and Environmental Health, vol. 78, no. 8, pp. 603612, 2005.

[105] U. J. Schlegel, M. Sturm, V. Ewerbeck, and S. J. Breusch, "Efficacy of vacuum bone cement mixing systems in reducing methylmethacrylate fume exposure: comparison of 7 different mixing devices and handmixing," Acta Orthopaedica Scandinavica, vol. 75, no. 5, pp. 559-566, 2004.

[106] A. W. Elmaraghy, B. Humeniuk, G. I. Anderson, E. H. Schemitsch, and R. R. Richards, "The role of methylmethacrylate monomer in the formation and haemodynamic outcome of pulmonary fat emboli," The Journal of Bone and Joint SurgeryBritish Volume, vol. 80, no. 1, pp. 156-161, 1998.

[107] K. Klemm, S. L. Henry, and D. Seligson, "The treatment of infection after interlocking nailing," Techniques in Orthopaedics, vol. 3, no. 3, pp. 54-61, 1988.

[108] S. C. Tandon and P. B. M. Thomas, "Persistent osteomyelitis of the femur- 2 cases of exchange intramedullary nailing with gentamicin beads in the nail," Acta Orthopaedica Scandinavica, vol. 67, no. 6, pp. 620-622, 1996.

[109] K. Anagnostakos and K. Schröder, "Antibiotic-impregnated bone grafts in orthopaedic and trauma surgery: a systematic review of the literature," International Journal of Biomaterials, vol. 2012, Article ID 538061, 9 pages, 2012.

[110] T. Fuchs, R. Stange, G. Schmidmaier, and M. J. Raschke, “The use of gentamicin-coated nails in the tibia: preliminary results of a prospective study," Archives of Orthopaedic and Trauma Surgery, vol. 131, no. 10, pp. 1419-1425, 2011.

[111] L. Legout and E. Senneville, "Periprosthetic joint infections: clinical and bench research," The Scientific World Journal, vol. 2013, Article ID 549091, 17 pages, 2013.

[112] C. G. Zalavras and M. Sirkin, "Treatment of long bone intramedullary infection using the RIA for removal of infected tissue: indications, method and clinical results," Injury, vol. 41, no. 2, pp. S43-S47, 2010. 
[113] J. C. Carmen, J. L. Nelson, B. L. Beckstead et al., "Ultrasonicenhanced gentamicin transport through colony biofilms of Pseudomonas aeruginosa and Escherichia coli," Journal of Infection and Chemotherapy, vol. 10, no. 4, pp. 193-199, 2004.

[114] T. Lin, X.-Z. Cai, M.-M. Shi et al., "In vitro and in vivo evaluation of vancomycin-loaded PMMA cement in combination with ultrasound and microbubbles-mediated ultrasound," BioMed Research International, vol. 2015, Article ID 309739, 7 pages, 2015. 

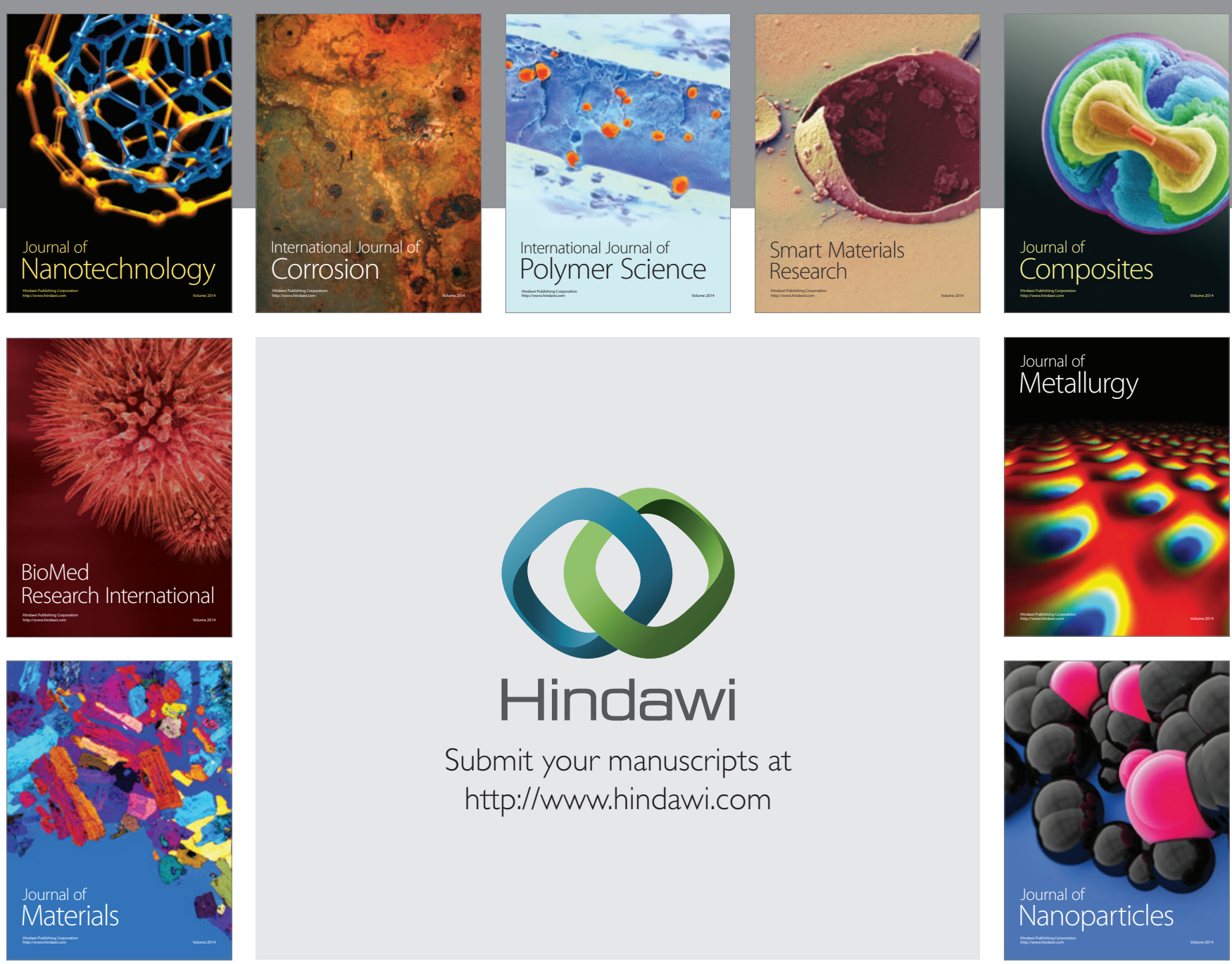

Submit your manuscripts at http://www.hindawi.com
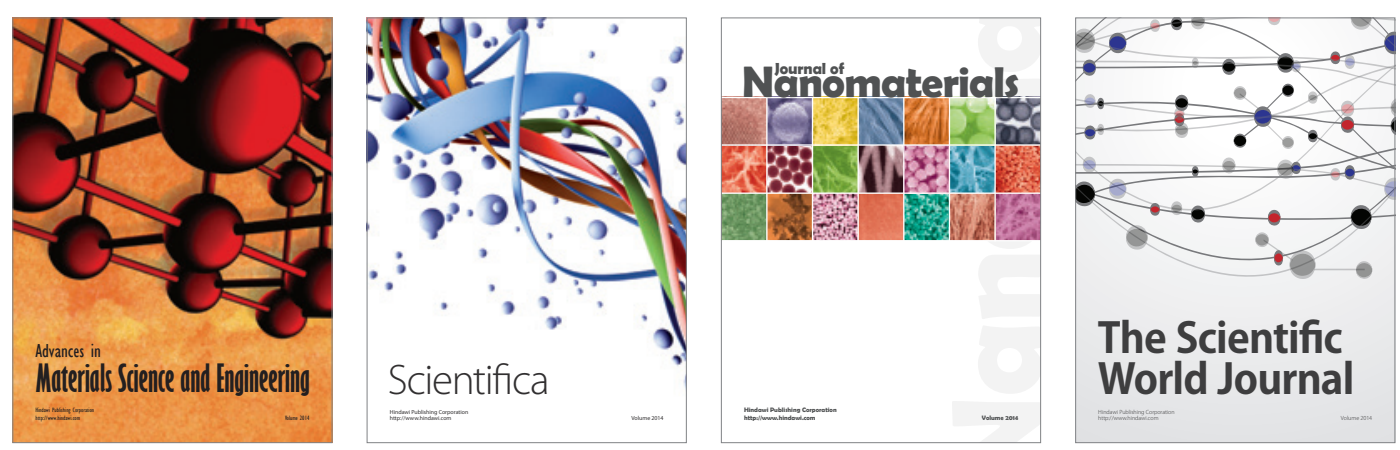

\section{The Scientific World Journal}
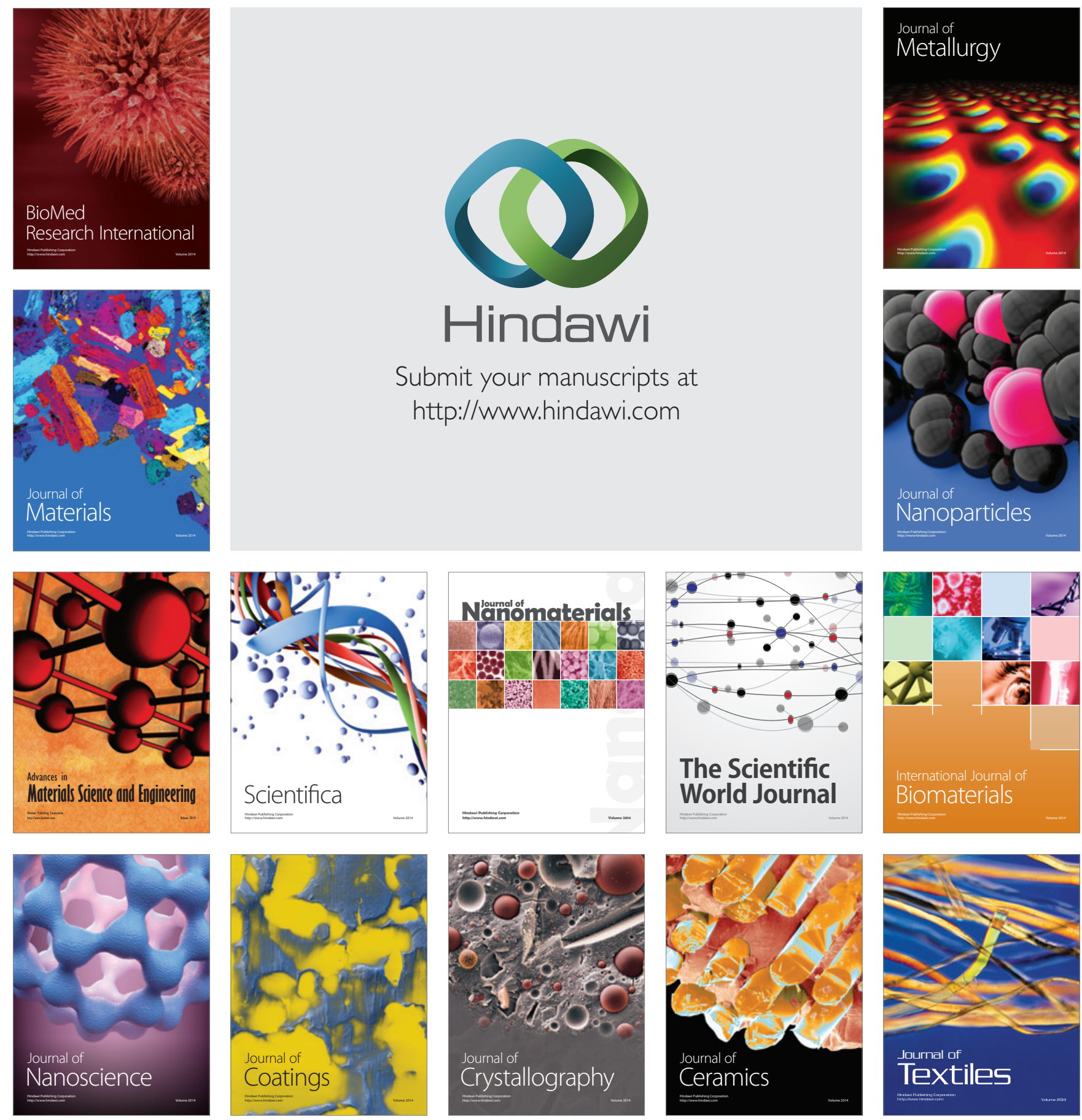